\title{
Comparison of Quality of Life of Patients with Hydrocele and Quality of Life of Patients with Inguinal Hernia
}

\author{
Seneviratne R. W. ${ }^{1}$, Kumara M. M. A. J. ${ }^{1}$, Abewickrama R. ${ }^{1}$, Kumarasinghe J. P. M. ${ }^{1}$, \\ Somasiri K. G. ${ }^{2}$, De Silva P. V. ${ }^{3}$ \\ ${ }^{1}$ Department of Surgery, Faculty of Medicine, University of Ruhuna, Galle, Sri Lanka \\ ${ }^{2}$ Department of Physiology, Faculty of Medicine, University of Ruhuna, Galle, Sri Lanka \\ ${ }^{3}$ Department of community Medicine, Faculty of Medicine, University of Ruhuna, Galle, Sri Lanka
}

Email address:

ranjanamst@yahoo.com (Seneviratne R. W.)

\section{To cite this article:}

Seneviratne R. W., Kumara M. M. A. J., Abewickrama R., Kumarasinghe J. P. M., Somasiri K. G., De Silva P. V.. Comparison of Quality of Life of Patients with Hydrocele and Quality of Life of Patients with Inguinal Hernia. European Journal of Preventive Medicine. Special Issue: New Frontiers of Public Health from the Pearl of Indian Ocean, Sri Lanka. Vol. 3, No. 2-1, 2015, pp. 47-49.

doi: $10.11648 /$ j.ejpm.s.2015030201.18

\begin{abstract}
Introduction: Quality of life (QOL) is one measure of how much a disease has affected the life of a patient from his or her perspective. WHOQL 100 assesses QOL under six domains and 24 facets along with overall quality of life. It was translated to Sinhala and was validated. QOL of hydrocele patients as well as inguinal hernia patients is expected to be lower than normal. Method: Ethical clearance was obtained from the ethical review committee of the Faculty of Medicine, University of Ruhuna, WHOQOL 100 was self administered to patients admitted for hydrocelectomy or herniotomy before surgery and by-standers in surgical ward as the control. Results: There were 23 males subjects in each category. The mean ages and SDs were $43.8 \pm 12.9$ (Hydrocele), $44.4 \pm 14.0$ (Control) and 43.7 \pm 11.9 (Hernia). QOL score of physical capacity (66.4 \pm 9.7 and $78.0 \pm 12.1 ; \mathrm{p}=0.001)$, psychological $(65.5 \pm 15.4$ and $78.0 \pm 9.5 ; \mathrm{p}=0.002)$ and level of independence $(67.7 \pm 14.3$ and $76.5 \pm 12.7$; $\mathrm{p}=0.033$ ) domains of hydrocele patients were significantly lower than those of the control. QOL scores of social relation were significantly lower in hydrocele patients than in hernia patients $(60.1 \pm 15.5$ and $71.5 \pm 14.4 ; \mathrm{p}=0.014)$. Conclusions: QOL of hydrocele patients were lower than normal. Physical, psychological and level of independence are more affected than the other aspects of QOL. Psychological aspects of the QOL are lower in hydrocele patients than in patients with hernia. Assessment of QOL using WHOQOL 100 can be considered as a valid tool to find out the impact of hydrocele on different aspects health.
\end{abstract}

Keywords: Quality of life, Hydrocele, Inguinal hernia

\section{Introduction}

Quality of life (QOL) is one measure of how much a disease has affected the life of a patient from his or her perspective. There are many instruments developed to measure QOL. Some instruments are disease specific and some others are generic. WHOQOL 100 is a generic instrument developed by World Health Organization to measure QOL of patients. WHOQL 100 is translated and validated to Sinhala. It assesses QOL under six domains, 24 facets and overall quality of life. The domains that assesses in WHOQOL 100 are physical capacity, psychological, level of independence, social relationship, environment and spirituality.

Hydrocele is commoner in geographical areas where filariasis is endemic. Hydrocele is a well known complication of lymphatic filariasis. There is accumulation of fluid in tunica vaginalis leading to enlargement of one or both scrotal sacs. Studies done to find QOL of hydrocele patients are scarce. Harichandrkumar et al studied QOL of lymphatic filariasis with or without hydroceIe using EuroQol and it was found that all domains QOL were affected in patients with hydrocele (1). Bontha et al have shown that there was impairment of sexual functions, lack of sexual satisfaction, loss of work capacity and hampered economic situation in patients with hydrocele (2). As quoted by David G Addiss and Molly A Brady in their review article there was a reduction in mobility, inability to perform certain daily activities as well as frustration in patients with hydrocele (3). Wickrmasinghe et al found that QOL scores were lower than 
normal even in patients who have undergone hydrocelectomy. Ahorlu et al have shown that there was improvement in work capacity, sexual functions and self esteem between 3-6 months after surgery (4).

Inguinal hernia is a common surgical condition encountered in most populations. Yet there is paucity of evidence on comparison of QOL between healthy individuals and patients with inguinal hernia. There are few publications on effect of surgical treatment on quality of life. There are no studies done using WHOQOL to assess QOL in patients with inguinal hernia. Kate Lawrence et al have compared QOL of pre and post surgical hernia patients with established population norms (5). Changes in QOL after surgical repair of inguinal hernia were assessed using generic instruments such as RAND-36 and SF $36(5,6,7,8)$. Carolina comfort scale is a specific instrument that was developed to assess post surgical condition of hernia patient (9). Though some QOL scores improved significantly after one month of surgery, they did not return to normal levels at the end of one month (10). QOL has improved significantly three months after repair of inguinal hernia as assessed by SF 36 (5). It was shown that QOL improves after surgical repair and it is comparable to normal in six months after surgery (5).

Both conditions; inguinal hernia and hydrocele occurs in relation to genital region. Swelling in inguinal hernia is intermittent and swelling in hydrocele is continuously present

\section{Method}

Ethical clearance was obtained from ethical review committee of the Faculty of Medicine, University of Ruhuna, Sri Lanka to assess QOL of life in hydrocele patients in 2012 and to assess quality of life of hernia patients in 2013. WHOQOL 100 was self-administered to patients with hydrocele admitted to surgical a ward in a tertiary care hospital in the Southern province of Sri Lanka for hydrocelectomy in 2012. Similarly same questionnaire was self-administered to patients with inguinal hernia admitted to the same surgical ward for surgical repair in 2013. Data collection was done prior to surgical procedure in both conditions. A control was taken from male by-standers in the surgical ward in 2013.

There were 23 scrotal hydrocele patients recruited for QOL assessment. Data from 23 age matched male inguinal hernia and male controls were taken for the statistical analysis. Independent t-test was done to compare QOL scores of hydrocele patients with the control as well as QOL scores of hydrocele patients with QOL scores of inguinal hernia patients.

\section{Results}

There were 23 subjects in each category. The mean age, $\mathrm{SD}$ and age range is given in table 1 . All subjects were males.

Table 1. Age distribution.

\begin{tabular}{lll}
\hline & Mean and SD & Range \\
\hline Hydrocele & $43.8 \pm 12.9$ & $23-69$ \\
Control & $44.4 \pm 14.0$ & $20-73$ \\
Hernia & $43.7 \pm 11.9$ & $22-72$ \\
\hline
\end{tabular}

Table 2 shows mean QOL scores and SD for different facets and domains of hydrocele patients, male control and male patients with inguinal hernia. It also gives $p$ values for QOL comparison between hydrocele with control and QOL comparison between hydrocele with hernia.

QOL score shows a pattern especially in domains physical capacity, psychological and level of independence as well as most of their facets. The patterns in QOL score are lowest in patients with hydrocele, highest in control group and inguinal hernia falls in between. The pattern is not obvious in other domains; social relationship, environment and spirituality.

Comparison of QOL scores of hydrocele with QOL scores of control group shows that hydrocele patients have significantly lower values for domain physical capacity, psychological, level of independence and their facets. Domain psychological and their facets of QOL scores were significantly lower in hydrocele patients than in hernia patients.

Table 2. Mean QOL scores between hydrocele control with $p$ value and $Q O L$ scores hydrocele between hernia with $p$ value.

\begin{tabular}{|c|c|c|c|c|c|}
\hline Domain or facet & $\begin{array}{l}\text { Hydrocele } \\
\text { mean } \pm \text { SD }\end{array}$ & $\begin{array}{l}\text { Control } \\
\text { mean } \pm \text { SD }\end{array}$ & $\mathbf{p}$ & hernia & $\begin{array}{l}\text { Phydrocele vs. } \\
\text { hernia }\end{array}$ \\
\hline Domain I Physical Capacity & $66.4 \pm 9.7$ & $78.0 \pm 12.1$ & 0.001 & $69.7 \pm 16.6$ & 0.419 \\
\hline 1 Pain and discomfort & $36.4 \pm 11.3$ & $20.7 \pm 14.3$ & 000 & $32.1 \pm 19.2$ & 0.353 \\
\hline 2 Energy and fatigue & $67.4 \pm 11.5$ & $74.5 \pm 16.3$ & 0.096 & $68.3 \pm 15.7$ & 0.812 \\
\hline 3 Sleep and rest & $68.2 \pm 18.6$ & $80.1 \pm 17.5$ & 0.031 & $72.0 \pm 22.1$ & 0.531 \\
\hline Domain II Psychological & $65.5 \pm 15.4$ & $78.0 \pm 9.5$ & 0.002 & $69.7 \pm 16.6$ & 0.419 \\
\hline 4 Positive feelings & $66.6 \pm 20.3$ & $80.2 \pm 16.7$ & 0.017 & $76.1 \pm 19.2$ & 0.110 \\
\hline 6 Self-esteem & $68.8 \pm 18.7$ & $75.8 \pm 15.9$ & 0.174 & $79.3 \pm 12.0$ & 0.027 \\
\hline 7 Bodily image and appearance & $68.6 \pm 19.1$ & $84.0 \pm 14.0$ & 0.003 & $83.4 \pm 18.3$ & 0.010 \\
\hline 8 Negative feelings & $36.4 \pm 17.0$ & $19.6 \pm 15.1$ & 0.001 & $26.4 \pm 18.1$ & 0.059 \\
\hline Domain III Level of Independence & $67.7 \pm 14.3$ & $76.5 \pm 12.7$ & 0.033 & $69.8 \pm 16.2$ & 0.650 \\
\hline 9 Mobility & $62.8 \pm 16.2$ & $75.0 \pm 17.7$ & 0.018 & $66.0 \pm 21.2$ & 0.561 \\
\hline 10 Activities of daily living & $67.7 \pm 16.5$ & $82.6 \pm 14.8$ & 0.002 & $76.8 \pm 18.7$ & 0.086 \\
\hline 11 Dependence on medication or treatments & $25.8 \pm 19.9$ & $25.3 \pm 15.8$ & 0.919 & $32.4 \pm 23.1$ & 0.304 \\
\hline
\end{tabular}




\begin{tabular}{|c|c|c|c|c|c|}
\hline 13 Personal relationships & $64.9 \pm 18.3$ & $72.5 \pm 15.5$ & 0.140 & $75.6 \pm 16.5$ & 0.043 \\
\hline 14 Social support & $56.0 \pm 18.3$ & $58.5 \pm 20.2$ & 0.658 & $64.5 \pm 20.8$ & 0.152 \\
\hline 15 Sexual activity & $59.5 \pm 17.6$ & $73.0 \pm 20.5$ & 0.023 & $74.3 \pm 19.6$ & 0.010 \\
\hline Domain V Environment & $56.8 \pm 15.9$ & $64.1 \pm 10.3$ & 0.186 & $64.5 \pm 14.2$ & 0.207 \\
\hline 16 Physical safety and security & $64.7 \pm 18.8$ & $70.7 \pm 15.4$ & 0.244 & $73.4 \pm 15.8$ & 0.097 \\
\hline 17 Home environment & $68.2 \pm 20.9$ & $77.2 \pm 14.9$ & 0.101 & $78.3 \pm 19.2$ & 0.096 \\
\hline 18 Financial resources & $46.5 \pm 23.5$ & $53.0 \pm 18.3$ & 0.299 & $54.5 \pm 22.1$ & 0.237 \\
\hline 19 Health and social care: accessibility and quality & $59.8 \pm 18.6$ & $54.1 \pm 14.7$ & 0.255 & $63.3 \pm 19.1$ & 0.528 \\
\hline 21 Participation in and opportunities for recreation/ leisure activities & $55.2 \pm 18.4$ & $63.6 \pm 16.1$ & 0.106 & $67.0 \pm 19.1$ & 0.040 \\
\hline 22 Physical environment (pollution/noise/traffic/climate) & $67.1 \pm 17.4$ & $74.7 \pm 15.7$ & 0.127 & $67.9 \pm 17.6$ & 0.882 \\
\hline 23 Transport & $60.3 \pm 24.5$ & $66.4 \pm 18.9$ & 0.318 & $59.1 \pm 28.3$ & 0.876 \\
\hline Domain VI Spirituality/Religion/Personal Beliefs & $60.9 \pm 16.6$ & $63.6 \pm 19.7$ & 0.861 & $63.6 \pm 19.7$ & 0.598 \\
\hline Overall quality of life and general health perceptions & $63.6 \pm 19.7$ & $72.6 \pm 16.5$ & 0.102 & $73.3 \pm 18.3$ & 0.095 \\
\hline
\end{tabular}

\section{Discussion}

Results of the study indicates that QOL scores of hydrocele patients are lower than normal when assessed by the WHOQOL-100 with special reference to physical capacity, psychological and level of independence domains. There were no studies done in the past using the WHOQOL100 to assess QOL of pre surgical hydrocele patients. Results of the study done by Harichandrkumar et al using EuroQOL had shown that all domains were affected in patients with hydrocele (1). Bontha et al has reported loss of work capacity as assessed by focus group discussions (2).

There were few studies done by conducting focus group discussions to assess the impact of hydrocele on different aspects of health. Comparison of the results of those studies with our results indicate similar pattern. Our results show that the score for sexual activity is significantly lower in hydrocele patients than that in control group as well as in patients with inguinal hernia. It is comparable to findings of Bontha et al (2). Bontha et al have also reported hampered economic situation in hydrocele patients (2). Though our results indicate the facet score for financial resources are lower in hydrocele patients than the control the difference is not significant.

As quoted by David G Addiss and Molly A Brady in review article there was a reduction in mobility, unable to perform certain daily activities, frustration in patients with hydrocele (3). Our results also show a similar pattern that is the scores for psychological domain and its facets are significantly lower than the control and hernia patients. Similarly the scores for facets mobility and daily living activities of hydrocele patients are significantly lower in hydrocele patients than in the control.

The comparison of QOL scores of domain psychological and social relations shows significantly lower values for hydrocele patients than in inguinal hernia patients. The results of QOL as assessed by WHOQOL are comparable to findings of focus group discussions (2).

\section{Conclusions}

QOL of hydrocele patients are lower than normal. Physical, psychological and level of independence is more affected than the other aspects of QOL. Psychological aspects of the QOL are lower in hydrocele patients than in patients with hernia. Assessment of QOL using WHOQOL 100 is valid to find the impact of hydrocele on different aspects health.

\section{References}

[1] Harichandrakumar KT, Krishnamoorthy K, Kumari AK, Das LK. Health status of lymphatic filariasis assessed from patients using seven domains five levels (7D5L) instrument. Acta Tropica. 2006 Oct: 99(2-3):137-43.

[2] Bontha V, Babu, Suchismita M, Abahara N. Nayak. Marriage, Sex and Hydrocele: An Ethnographic Study on the Effect of Filarial Hydrocele on Conjugal Life and Marriage Ability from Orissa, India. Neglected tropical diseases. 2009:3:e414.

[3] David GA, Molly AB. Morbidity management in the Global Programme to Eliminate Lymphatic Filariasis. Filaria Journal.2007: 6(2).

[4] Ahorlu CK, Dunyo SK, Asamoah G and Simonsen PE. Consequences of hydrocele and the benefits of hydrocelectomy a qualitative study in lymphatic filriasis endemic communities on the coast of Ghana. Acta Tropica. 2001 Dec 21:80(3):21521.

[5] Kate L, Crispin J, Douglas MW, Angela C. Quality of life in patients undergoing inguinal hernia repair. Ann R Coll Surg Engl. 1997:79: 40-45.

[6] Bitzer EM, Lorenz C, Nickel S et al. Patient-reported outcomes in hernia repair. Hernia. 2008 Aug: 12(4):407-14.

[7] Fei L, Filippone G, Trapani V et al. Feasibility of primary inguinal hernia repair with a new mesh. World J Surg. 2006 Jun: 30(6):1055-62.

[8] Pierides G, Mattila K, Vironen J. Quality of life change in elderly patients undergoing open inguinal hernia repair. Hernia. 2013 Dec: 17(6):729-36.

[9] Williams KB, Bradley JF, Wormer BA et al. Postoperative quality of life after open transinguinal preperitoneal inguinal hernia repair using memory ring or three-dimensional devices. AMJ Surg. 2013 Aug: 79(8):786-93.

[10] Kristiina M, Merja L, Markku H. Health-related quality of life following ambulatory surgery procedures: assessment by RAND-36. BMC Anesthesiology. 2012. 